\title{
Star-shaped hexaaryltriindoles small molecules: Tuning molecular properties towards solution processed organic light emitting devices
}

\author{
Carmen Coya ${ }^{\mathrm{a}, *}$, Constanza Ruiz ${ }^{\mathrm{b}}$, Ángel Luis Álvarez a , Susana Álvarez-García a,c, \\ Eva M. García-Frutos ${ }^{\mathrm{b}}$, Berta Gómez-Lor ${ }^{\mathrm{b}, *}$, Alicia de Andrés ${ }^{\mathrm{b}, *}$ \\ ${ }^{a}$ Escuela Superior de Ciencias Experimentales y Tecnología, Electronic Technology Department, Universidad Rey Juan Carlos, 28933 Madrid, Spain \\ ${ }^{\mathrm{b}}$ Instituto de Ciencia de Materiales de Madrid, Cantoblanco, 28049 Madrid, Spain \\ ' Instituto Química-Física Rocasolano, CSIC, C/Serrano 119, 28006 Madrid, Spain
}

\section{A R T I C L E I N F O}

\section{Article history:}

Received 20 February 2012

Received in revised form 14 June 2012

Accepted 15 June 2012

Available online 28 June 2012

\section{Keywords:}

Solution processing

Molecular OLED

Blue emitters

\begin{abstract}
A B S T R A C T
We present a series of differently substituted star-shaped hexaaryltriindoles with tunable light-emitting properties. The deep blue emission is unchanged by donor peripheral substituents while an increasing acceptor character produces a reduction of the optical gap, an increased Stokes shift and eventually leads to the appearance of a new electronic level and to the simultaneous deep blue $(413 \mathrm{~nm})$ and green $(552 \mathrm{~nm})$ emission in solution. Quenching by concentration increases with the acceptor character but is lower as the tendency of these compounds to aggregate is stronger. Solution processed thin films present optical and morphological qualities adequate for device fabrication and similar electronic structure compared to solutions with an emission range from $423 \mathrm{~nm}$ up to $657 \mathrm{~nm}$ (red), demonstrating the possibility of tuning the energy levels by chemical functionalization. We have fabricated and characterized single-layer solution processed organic light emitting diodes (OLED) to investigate the influence on transport and emission properties of the substituting species. We analyzed the $I-V$ response using a single-carrier numerical model that includes injection barriers and non-uniform electric-field across the layer. As a result, we obtained the electric field dependence of the mobility for each device. Best results are obtained on the most electron rich derivative functionalized with six donor methoxy groups. This material shows the highest emission efficiency in solid state, due to aggregation-induced enhancement, and better transport properties with the highest mobility and a very low turn-on voltage of $2.8 \mathrm{~V}$. The solution processed OLED devices produce stable deep blue (CIE coordinates $(0.16,0.16)$ ) to white (CIE coordinates $(0.33,0.3)$ ) emission with similar luminous efficiencies.
\end{abstract}

(c) 2012 Elsevier B.V. All rights reserved.

\section{Introduction}

In recent years an intense research effort has facilitated that a new generation of devices based on organic materials enters the market. Organic semiconductors offer great expectations in the development of low-cost, flexible and largearea devices (properties difficult to achieve with conven-

\footnotetext{
* Corresponding authors.

E-mail address: ada@icmm.csic.es (A. de Andrés).
}

tional electronics) but also the possibility of fine tuning key properties (luminescence, absorption, energy band gaps, and charge transport) through a convenient molecular design. A wide variety of polymers and small molecule organic semiconductors have been developed and investigated in devices such as organic thin-film transistors (OTFTs), organic photovoltaic cells (OPVs), and organic light-emitting diodes (OLEDs).

Amenability to solution or wet processing, is an inevitable prerequisite for large area, low cost mass production, and must be taken into consideration when designing mol- 
ecules for organic active layers [1]. Conjugated polymers, usually amorphous materials can be easily processed from solution to yield homogeneous and uniform thin films, ideally suited for optoelectronics. However, small molecule semiconductors offer intrinsic advantages over polymeric materials in terms of ease of synthesis and purification. Owing to their monodisperse nature with well-defined chemical structure, they can be reproducibly prepared, functionalized and purified and batch-to batch variations common in polymeric materials are not a problem. These considerations make small molecules ideal candidates for structure-properties studies.

Incorporation of flexible alkyl chains is a useful strategy for enhancing the solubility of small molecules, facilitating wet processing. On the other hand, incorporation of bulky substituents able to promote various conformers, have successfully prevented easy packing of the molecules and hence ready crystallization resulting in excellent filmforming abilities and morphological stability in properly designed molecules. We have recently became interested in new organic semiconductor, the electron-rich $10,15-$ dihydro-5H-diindolo[3,2-a:3', $2^{\prime}$-c]carbazole (triindole). Triindole-based single crystalline [2] or liquid crystalline materials have been found to exhibit high hole mobilities $[3,4]$ since they combine the good hole transport properties of carbazoles with a high tendency to form columnar supramolecular arrangements owing to their disk-like extended $\pi$-surface. Devices, such as OFETs [5] and OLEDs [6] have been fabricated using triindole-based semiconductors.

In addition, this platform provides two different locations for structural modification, essential to tailor electronic and self-assembling properties. Nitrogen can be easily $N$-substituted, while starting from known hexabromine triindol, the exploitation of cross-coupling methodologies opens flexible strategies to various functionalizations. Taking advantage of this chemical versatility we have introduced in this platform flexible $N$-alkyl chains intended to induce solubility (long alkylic chains) and peripheral bulky aryl groups that impede crystallization [7] to render a family of star-shaped hexaaryl triindoles that can be easily processed from solution to give good film-forming properties. A study of the electronic character of the peripheral substituents on the self-association behavior in these series indicates that the electron-donating character of the terminal substituents facilitates self-association while electronwithdrawing groups inhibit aggregation in agreement with important contributions of solvophobic and $\mathrm{CH}-\pi$ interactions in the self-assembly process. In fact attaching six strong donor methoxy groups result in a material that is liquid crystals in a broad range of temperature [8].

In this work, we show how the electronic communication of the external substituents with the central electron-rich triindole facilitates the tuning of the light-emitting properties and HOMO-LUMO levels. We study these small molecular materials in amorphous thin film morphology and we report emission and transport properties of solution processed OLEDs based on three representative triindole derivatives. The fabricated OLEDs have simple structure: glass/ indium-tin oxide (ITO)/poly(3,4-ethylenedioxythiophene)poly(styrenesulfonate) (PEDOT:PSS)/active layer/Ca/Al. The devices allow us to study the current-voltage $(I-V)$ response using a numerical model that includes fielddependent carrier mobility under a single carrier approach. The mobility dependence with electric field and the electroluminescence (EL) performance is discussed.

\section{Experimental}

2.1. Synthesis and characterization of 2,3,7,8,12,13-Hexakis(p-formylphenyl)-5,10,15-tris-(dodecyl)-10,15-dihydro-5Hdiindolo[3,2-a:3',2'-c]carbazole (1e)

A mixture of $\mathrm{N}$-dodecylhexabromotriindole [9] (100 mg, $0.075 \mathrm{mmol}), \mathrm{Pd}\left(\mathrm{PPh}_{3}\right)_{4}(43 \mathrm{mg}, 0.037 \mathrm{mmol})$, 4-formylphenyl boronic acid (136 mg, $0.907 \mathrm{mmol}$ ) in $0.5 \mathrm{~mL}$ of $2 \mathrm{M}$ aqueous $\mathrm{K}_{2} \mathrm{CO}_{3}$ and $4 \mathrm{~mL}$ of THF was degassed. The mixture was heated at $90^{\circ} \mathrm{C}$ for 4 days under nitrogen. The orange suspension was partitioned between $\mathrm{H}_{2} \mathrm{O}$ and $\mathrm{CH}_{2} \mathrm{Cl}_{2}$, and the organic phase dried $\left(\mathrm{MgSO}_{4}\right)$. The solvent was evaporated and the residue was precipitated with acetone and acetonitrile to give 1e as a yellow solid (78 mg, 70\%): mp:173-176 ${ }^{\circ} \mathrm{C},{ }^{1} \mathrm{H}$ NMR (200 MHz, $\left.\mathrm{CDCl}_{3}\right) \delta 10.02$ $(\mathrm{s}, 6 \mathrm{H}), 8.37(\mathrm{~s}, 3 \mathrm{H}), 7.82(\mathrm{~d}, J=8.0,12 \mathrm{H}), 7.68(\mathrm{~s}, 3 \mathrm{H})$, $7.46(\mathrm{~d}, J=8.0,12 \mathrm{H}), 4.99(\mathrm{~m}, 6 \mathrm{H}), 2.10(\mathrm{~m}, 6 \mathrm{H}), 1.05(\mathrm{~m}$, $54 \mathrm{H}), 0.85(\mathrm{t}, J=6.7,9 \mathrm{H}) ;{ }^{13} \mathrm{C} \mathrm{NMR}\left(50 \mathrm{MHz}, \mathrm{CDCl}_{3}\right) \delta$ $191.8,191.7,148.7,148.10,140.8,140.0,134.8,134.6$, $134.4,132.0,131.0,130.8,129.6,123.6,123.2,112.2$, 103.2, 47.4, 31.8, 30.3, 29.6, 29.5, 29.4, 29.3, 26.7, 22.6, 14.1; UV $\left(\mathrm{CH}_{2} \mathrm{Cl}_{2}, 25^{\circ} \mathrm{C}\right) \lambda_{\max }(\log \varepsilon) 361$ (4.99); MALDITOF MS $m / z 1475\left(\mathrm{M}^{+}\right)$; HRMS (MALDI-TOF) calcd. for $\mathrm{C}_{102} \mathrm{H}_{111} \mathrm{~N}_{3} \mathrm{O}_{6}$ : 1473.84674 , found: 1473.84387 .

\subsection{Synthesis and characterization of 2,3,7,8,12,13-Hexakis- (p-dicyanomethyl)-5,10,15-tris-(dodecyl)-10,15-dihydro-5H- diindolo[3,2-a:3',2'-c]carbazole( $1 f)$}

To a mixture of 1e (100 mg, $0.067 \mathrm{mmol})$, malononitrile (53.5 mg, $0.435 \mathrm{mmol})$, pyridine $(0.13 \mathrm{~mL}, 1.62 \mathrm{mmol})$ and $\mathrm{CH}_{2} \mathrm{Cl}_{2}(20 \mathrm{~mL})$, titanium (IV) chloride $(0.11 \mathrm{~mL}, 0.81 \mathrm{mmol})$ was added. The mixture reaction mixture was stirred overnight at room temperature. The suspension was partitioned between $\mathrm{H}_{2} \mathrm{O}$ and $\mathrm{CH}_{2} \mathrm{Cl}_{2}$, and the organic phase dried $\left(\mathrm{MgSO}_{4}\right)$. The solvent was evaporated and the residue was washed with $\mathrm{MeOH}$ to yield $\mathbf{1 f}$ as a red solid (87 $\mathrm{mg}, 74 \%$ ): ${ }^{1} \mathrm{H} \mathrm{NMR}\left(200 \mathrm{MHz}, \mathrm{CDCl}_{3}\right) \delta 8.35(\mathrm{~s}, 6 \mathrm{H}), 7.89(\mathrm{~d}, J=7.5 \mathrm{H}$, $12 \mathrm{H},), 7.69(\mathrm{~s}, 3 \mathrm{H}), 7.48(\mathrm{~s}, 3 \mathrm{H}), 7.46(\mathrm{~d}, J=7.5 \mathrm{H}, 12 \mathrm{H}), 5.00$ $(\mathrm{m}, 6 \mathrm{H}), 2,08(\mathrm{~m}, 6 \mathrm{H}), 1,21(\mathrm{~m}, 54 \mathrm{H}) .,{ }^{13} \mathrm{C}$ NMR $(50 \mathrm{MHz}$, $\left.\mathrm{CDCl}_{3}\right) \delta$ 158.9, 153.6, 148.8, 148.1, 141.0, 140.3, $134.3,131.38,130.8,129.5,129.2,123.9,123.6,113.7$, 112.8, 112.5, 103.3, 82.5, 82.3,47.4, 31.9, 29.6, 29.3, 26.7, 22.6, 14.1, UV $\left(\mathrm{CH}_{2} \mathrm{Cl}_{2}, 25^{\circ} \mathrm{C}\right) \lambda_{\max }(\log \varepsilon) 230$ (5.25), 338 (5.55), 434 (5.24); MALDI-TOF MS m/z $1763\left(\mathrm{M}^{+}\right)$; HRMS (MALDI-TOF) calcd. for $\mathrm{C}_{102} \mathrm{H}_{111} \mathrm{~N}_{3} \mathrm{O}_{6}$ : 1762.9173, found: 1762.9134 .

\subsection{Optical measurements}

Optical absorption and photoluminescence (PL) spectra of the material in solution and in thin films on quartz substrates have been measured at room temperature. The absorption spectra were recorded with a Varian spectrophotometer 
model Cary 4000. PL was excited with the $365 \mathrm{~nm}$ line of a $\mathrm{Hg}$ lamp, and analyzed with a Jobin-Yvon HR 460 monochromator coupled to a $\mathrm{N}_{2}$ cooled charge coupled device. All the emission spectra have been corrected by the spectral response of the experimental setup. Emission lifetimes were recorder at room temperature exciting with an OPO laser from EKSPLA and an ultra-rapid intensified charge coupled device from Stanford Computer Optics. Spectral radiance and luminance from diodes was recorded with a Konica-Minolta CS-2000 spectroradiometer, in the same excitation conditions (duty cycle) as those used to measure $J-V$ characteristics (duty cycle of $0.2 \%$ ) using a TTi $40 \mathrm{MHz}$ arbitrary waveform generator and a TREK-601C amplifier.

\subsection{Device fabrication}

The structure of the devices are ITO/PEDOT:PSS (50 nm)/ Active layer/Cathode. Pre-patterned ITO glass plates with four circular diodes ( $1 \mathrm{~mm}$ and $1.5 \mathrm{~mm}$ radii) were extensively cleaned, using chemical and UV-ozone methods, just before the deposition of the organic layers. PEDOT:PSS (483095 from Aldrich) was deposited at $2000 \mathrm{rpm}$ by spin-coating and then cured on a hot plate at $110^{\circ} \mathrm{C}$ for $10-15 \mathrm{~min}$. The active layers were deposited by spin coating from chloroform solutions and cured at $50{ }^{\circ} \mathrm{C}$ for $30 \mathrm{~min}$ on a hot plate. Precursor solutions $(10 \mathrm{mg} / \mathrm{mL}$ ) were kept in an ultrasonic bath for $10 \mathrm{~min}$ and filtered through a $0.2 \mu$ m polytetrafluoroethylene(PTFE) syringe filter, prior to be spin coated. We have found that heating the sample just below the solvent boiling point results in an improved film uniformity (highly reflecting surfaces) [10]. The thickness of the layers was measured using an Alpha step 200 profilomenter (Tenkor Instruments). The cathode was thermally evaporated in an atmosphere of $8 \times 10^{-6}$ Torr on top of the organic layer surface and finally, the structure was encapsulated using a glass cover attached by a bead of epoxy adhesive [EPO-TEK(302-3M)]. All the process was carried out in an inert atmosphere glovebox $\left(<0.1 \mathrm{ppm} \mathrm{O}_{2}\right.$ and $\left.\mathrm{H}_{2} \mathrm{O}\right)$.

\subsection{Electrical measurements}

The device current-voltage characteristics were measured using a semiconductor parameter analyzer Agilent 4155C and a SMU pulse generator Agilent 41501B. A pulse train was used as input signal. The duty cycle was set to be $0.2 \%$, thus having a pulse width of $0.5 \mathrm{~ms}$ for a period of 100 ms. Refresh time between two consecutive pulses ensures long time operation without significant device degradation. Furthermore, the $I-V$ curve stability was achieved by gradually increasing the pulse amplitude up to the point where reproducible measurements were observed. The voltage range was maintained below the onset for the electroluminescence (EL).

\section{Results and discussion}

\subsection{Synthesis}

The synthesis of the hexaaryl triindoles 1a-1d Fig. 1 has been previously reported and was achieved using sixfold Suzuki coupling of known $\mathrm{N}$-dodecylhexabromotriindole with commercially available boronic acids substituted in para with groups of different electronic character [7]. Using the same cross-coupling conditions 1e was readily achieved by reaction of $\mathrm{N}$-dodecylhexabromotriindole with (4-formylphenyl) boronic acid. Knoevenagel reaction of 1e with malonitrile in the presence of $\mathrm{TiCl}_{4}$ gave rise to compound $\mathbf{1 f}$ as a deep red material in good yield.

\subsection{Characterization of the electronic properties of solutions and thin films}

The influence of the six peripheral groups, in the electronic properties of this family of compounds has been studied by cyclic voltammetry (Supporting information). All the compounds can be easily oxidized to stable radical cations and higher cationic charged species. The oxidazibility of these molecules varies in agreement with the nature of the peripheral substituents. Thus, attaching electrondonating groups results in an increase of the electron density in the $\pi$ system and in consequence in a shift of the oxidation potentials to lower values. In contrast, upon attaching peripheral electron-withdrawing groups the oxidation potentials shifts anodically as increasing the acceptor character of the substituents. Compound 1f show also a quasi reversible reduction wave in the accessible potential window of the solvent $\left(\mathrm{CH}_{2} \mathrm{Cl}_{2}\right)$.

The HOMO energy values for these derivatives (Table 1 ) have been estimated from the first oxidation potential values with respect to ferrocene value of $-4.8 \mathrm{eV}$ with respect to zero vacuum level. This value is obtained from the calculated value of $-4.6 \mathrm{eV}$ for the standard electrode potential $\left(E^{\circ}\right)$ using a normal hydrogen electrode (NHE) on the zero vacuum level and the value of $0.2 \mathrm{~V}$ for Fc vs. NHE.

The influence of the peripheral groups on the electronic properties of the differently substituted hexaaryltriindoles is also clearly reflected in their absorption and emission spectra. Fig. 2(a) and (b) compare the absorption and the emission spectra obtained at $365 \mathrm{~nm}$ excitation of 1a, 1d, 1e and 1f. Compounds 1a, $1 \mathbf{b}$ and 1c present almost identical absorption and emission spectra (shapes and intensities) while 1d and 1e show significant bathochromic shifts as the acceptor character of the peripheral groups increases. The emission of compound $\mathbf{1 f}$ is very weak (note the factor $\times 20$ in Fig. 2(b)) and presents two well separated

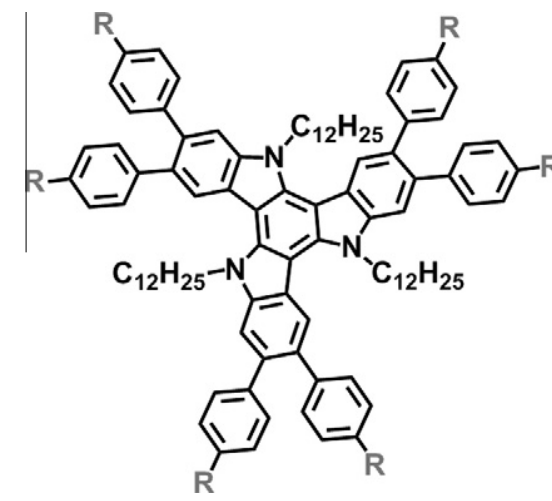


Table 1

Values of HOMO, lowest transition and absorption onset $E_{g}^{\text {onset }}$ (from absorbance) and main photoluminescence energies ( $E_{\mathrm{PL}}$ ) of compounds 1a-1f in solution and in thin films. All data are in $\mathrm{eV}$.

\begin{tabular}{|c|c|c|c|c|c|c|c|}
\hline \multirow[t]{2}{*}{$\mathrm{R}$} & \multicolumn{4}{|l|}{ Solutions } & \multicolumn{3}{|l|}{ Films } \\
\hline & НОМо & Lowest trans. & $E_{g}^{\text {onset }}$ & $E_{\mathrm{PL}}$ & Lowest trans. & $E_{g}^{\text {onset }}$ & $E_{\mathrm{PL}}$ \\
\hline $1 a$ & -5.07 & 3.17 & 3.0 & $3.08,2.98$ & 3.03 & 2.64 & 2.91 \\
\hline $1 b$ & -5.12 & 3.16 & 3.01 & $3.08,2.98$ & 3.07 & 2.66 & 2.91 \\
\hline $1 c$ & -5.12 & 3.17 & 3.03 & $3.08,2.98$ & 3.09 & 2.66 & 2.93 \\
\hline $1 d$ & -5.28 & 3.21 & 2.66 & 2.63 & 3.16 & 2.5 & 2.65 \\
\hline $1 \mathrm{e}$ & -5.32 & 3.15 & 2.55 & 2.32 & 3.07 & 2.5 & 2.56 \\
\hline 1f & -5.25 & 2.8 & 2.1 & $3.0,2.25$ & 2.66 & 1.96 & 1.89 \\
\hline
\end{tabular}
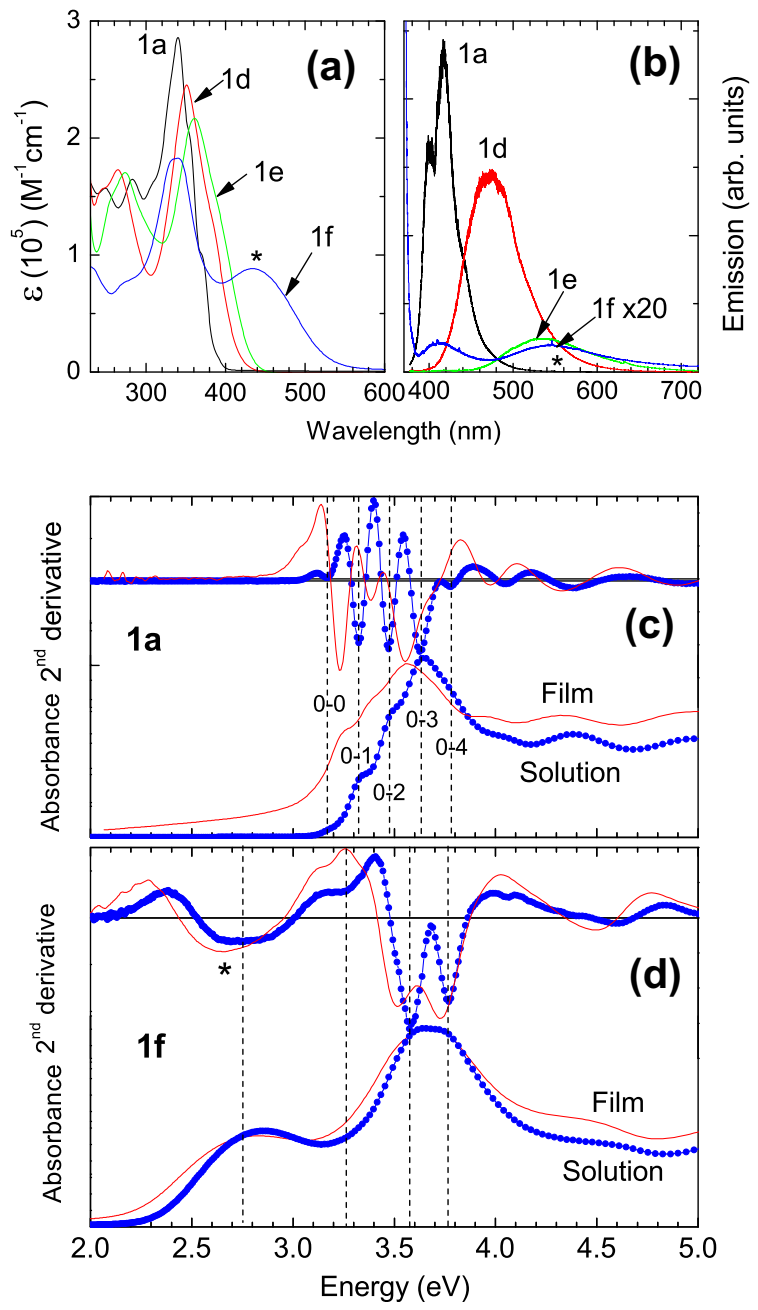

peaks at 413 and $552 \mathrm{~nm}$ (3.0 and $2.25 \mathrm{eV}$ ). The molar absorption coefficients are in the order of $10^{5}\left(\mathrm{M}^{-1} \mathrm{~cm}^{-1}\right)$ as it corresponds to $\pi-\pi^{*}$ transitions [11]. In Fig. 2(c) and (d) we compare the absorption spectra and their second derivatives for 1a and $\mathbf{1 f}$ compounds in solution and as thin films.
Second derivatives of absorbance are useful to evidence the different components of the spectra [12] (minima correspond to absorption maxima and therefore to electronic transitions). In Fig. 2(c), the vertical dashed lines correspond to transitions of $\mathbf{1 a}$ in solution. The five transitions (dashed vertical lines) of lower energy are equidistant in energy (around $151 \mathrm{meV}=1220 \mathrm{~cm}^{-1}$ ) and correspond to transitions from the ground state (HOMO) to different vibrational states of the first excited state (LUMO) labeled with ( $0-i)$ with $i=0$ to 4 . The lowest transition (0-0) occurs at $3.17 \mathrm{eV}$ while the main absorption peak is at $3.63 \mathrm{eV}$. This shift $(0.46 \mathrm{eV})$ reveals that the atomic configuration of the excited state is different from that corresponding to the ground state of the molecule. The observation of the vibrational levels allows identifying the 0-0 transition.

The absorption onset (departure from zero in the second derivatives of the absorption spectra) is often used to define the optical gap ( $3 \mathrm{eV}$, in this compound) since it is the minimum energy where an optical absorption occurs and corresponds to the transition between the upper and lower edges of the HOMO and LUMO bands, respectively. Since HOMO and LUMO are bands whose width is related to homogeneous but also to inhomogeneous broadening which has different origins as different environments, stacking, inter-molecular coupling, defects, etc., the gap calculated from the absorption onset is not an intrinsic parameter. On the contrary, the energies of the absorption peaks are closer to an intrinsic description of the electronic structure. Strictly speaking, the LUMO should be obtained using the (0-0) transition. Table 1 collects the observed "lowest transition" values, that correspond to the (0-0) transition in 1a to 1c, and that we used to obtain an estimation of the LUMO levels shown in Fig. 3(b). Table 1 also collects the optical gap, $E_{g}^{\text {onset }}$, corresponding to the absorption onset.

The energy of the main peak (stars) as well as that of the lowest detected transition for the different compounds (rhombi) are plotted in Fig. 3(a). The figure evidences that the energy of the main peak is almost unvaried except for 1d and 1e where the coupling to the acceptor groups produces a lowering of the main peak energy and the loss of the vibronic structure. As a result the gap is shifted to lower energies. But only the $\mathrm{CHC}(\mathrm{CN})_{2}$ groups, found in $\mathbf{1 f}$, which combine a strong acceptor character with an increase of the conjugation, lowers the gap down to about $2.1 \mathrm{eV}$ by means of a new transition (at $2.8 \mathrm{eV}$, indicated with an asterisk in Fig. 2(a) and a circle in Fig. 3(a)). This new electronic state is most probably related to the electronic density transferred 


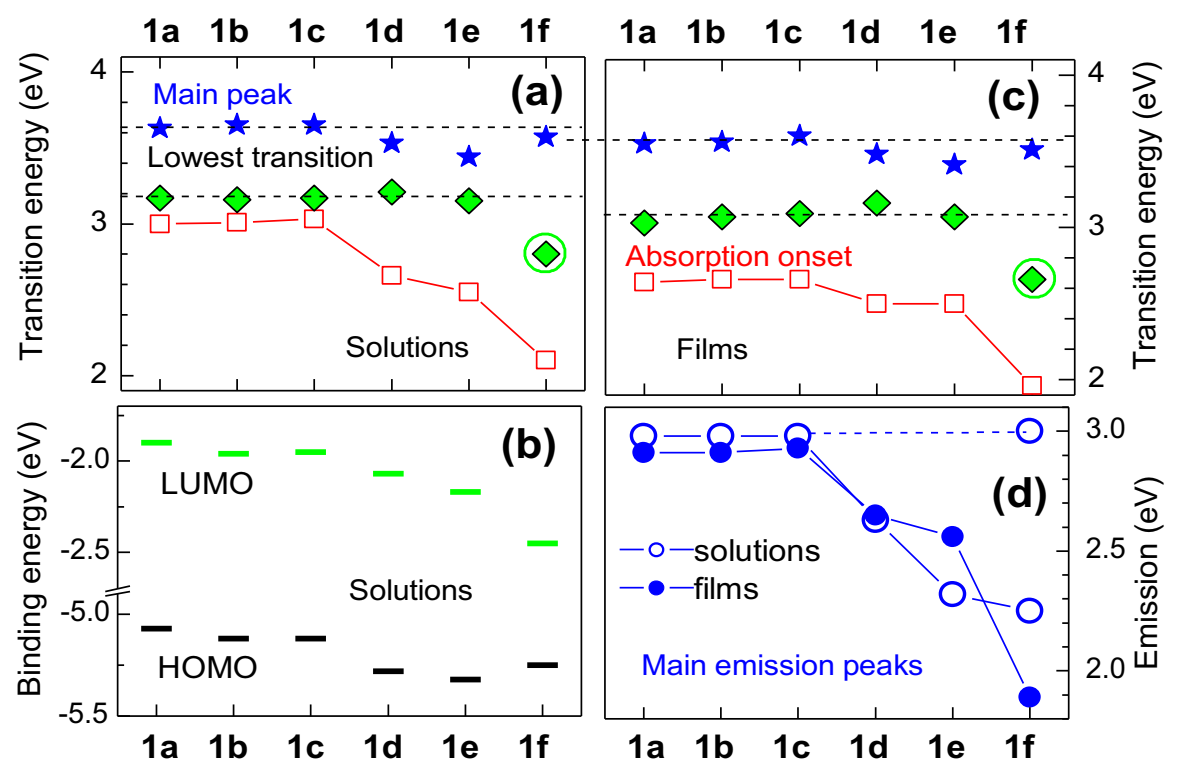

from the core to the peripheral groups giving rise to an octupolar character of the molecule. The progressive loss of the vibrational structure in $\mathbf{1 d}$ and $\mathbf{1 e}$ compounds prevents identifying the $(0-0)$ transition so that the lowest observed transition in these compounds are most probably related to the $(0-1)$ transition.

The dramatic influence that peripheral substituents of triindoles exert on the electronic properties of these derivatives offers a great opportunity to modulate their optical gap, again demonstrating the possibility of tuning the energy levels of these derivatives by chemical functionalization. Considering that organic electronic devices are usually constructed using multilayer architectures this possibility will be fundamental to facilitate charge injection from electrodes and minimize energy barriers between the different layers.

The emission spectra of $\mathbf{1 a}, \mathbf{1} \mathbf{b}$ and $\mathbf{1 c}$ compounds in solution are almost identical with two peaks at 403 and $418 \mathrm{~nm}$ (3.08 and $2.97 \mathrm{eV}$ ). In Fig. 2(b), the photoluminescence spectrum of 1a is shown as well as those corresponding to 1d, 1e and 1f. The emission spectra are consistent with the changes in the electronic structure, previously deduced from the absorption data, related to the different character of the peripheral groups. Time resolved spectroscopy experiments performed for 1a solution showed a lifetime of $3.0 \mathrm{~ns}$ for the $10^{-5} \mathrm{M}$ solution which is consistent with fluorescence relaxation from singlet excited state (see Supplementary Information). The red shift of the emission peaks of solutions of $\mathbf{1 d}$ and $\mathbf{1 e}$ increases as the acceptor character of the peripheral groups increases (Fig. 3(d) and Table 1). 1f emission in solution exhibits two distinct emission lines at 413 and $551 \mathrm{~nm}$ (3.0 and $2.25 \mathrm{eV}$, respectively). The highest energy peak, which coincides with that observed for $\mathbf{1 a - 1 c}$, corresponds

to the relaxation from the excited state corresponding to the "main peak" in Fig. 3(a) while the lower energy one $(2.25 \mathrm{eV})$ is the relaxation from the electronic level related to the charge transferred to the peripheral groups.

In order to select the best candidates for practical applications we have studied the film-forming properties of the new derivatives synthesized. Films of triindole derivatives were prepared by spin coating from $1 \mathrm{wt}$.\% dichloromethane or chlorobenzene as precursor solutions on quartz. The obtained films were highly transparent and uniform to the eye and the optical microscope images reveal no inhomogeneities. Films thickness ranges from 20 to $100 \mathrm{~nm}$.

The optical absorption spectra of the films, collected in Fig. 4(a), demonstrate their high optical quality and show almost identical features compared to the corresponding solution spectra with some differences in the peak energies (Table 1). The vibronic fine structure is also observed in thin films from 1a to 1c compounds maintaining the coupling of the excited state to the $150 \mathrm{meV}$ vibration (see 2nd derivative in Fig. 2(c)). The main absorption peaks correspond, as in the solutions, to the (0-3) transitions.

Figs. $3 \mathrm{~b}$ and $\mathrm{c}$ collect the energies of relevant electronic transitions from absorption and emission spectra of the thin films. A systematic shift to lower energies, in the range of $50-100 \mathrm{meV}$, is observed for the absorption bands of thin films compared to their dilute forms (Figs. 2 and 3). The lowering of the transition energies may be explained by the renormalization of the energy levels due to the close packing of the molecules. This energy shift is the only detected effect on the electronic structure related to the aggregation of the molecules in the thin films electronic transitions. The main emission of the thin films (Fig. 4(b)) ranges from the deep blue of the 1a-1c compounds (426 nm) to the red for $\mathbf{1 f}(656 \mathrm{~nm})$. It is therefore 


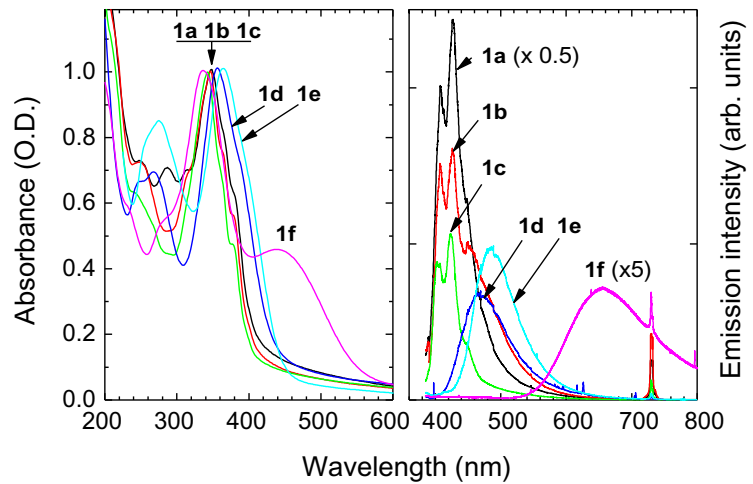

possible to tune the emission in the whole visible range by chemical functionalization.

It is well known that whereas many organic molecules emit strongly in solution, they become weak fluorophores when increasing the concentration or when forming thin films due to non-radiative relaxation processes as energy transfer between neighboring molecules. This is a common problem found when looking for molecules that have to be incorporated in devices. However, in conveniently functionalized molecules emission may be enhanced when increasing the concentration owing to the restriction of intramolecular rotation processes upon aggregation or from the inhibition of geometry relaxation of the vertical excited state to a non-emitting state [13].

We have performed a study of the fluorescence of these compounds at different concentrations and found that two mechanisms are relevant for the resulting external quantum efficiency. On one hand, the quenching by concentration increases as the electron withdrawing character of the peripheral groups increases (Fig. 5). Note that because of the high molar absorption coefficients $\varepsilon\left(\lambda_{\text {exc }}=365 \mathrm{~nm}\right)$ of these compounds, at concentrations of $10^{-5} \mathrm{M}$ the absorbed intensity $\left(I_{A} / I_{0}=1-\exp \left(-2.3 \varepsilon\left(\lambda_{\text {exc }}\right) l \mathrm{c}\right)=0.98-0.999\right.$, where $l=1 \mathrm{~cm}$ and $c$ is the concentration) is already almost saturated and, since the emission intensity is proportional to $I_{A}$, very weak or even no increase of the emission intensity is expected by increasing the concentration, as it occurs for $\mathbf{1 b}$ and $\mathbf{1 c}$ (Fig. 5). On the other hand, emission quenching by concentration is lower as the tendency of these compounds to aggregate is stronger, in particular an enhancement of the emission upon increasing the concentration is evident for 1a (Fig. 5). Apparently, in these triindole derivatives functionalized with bulky peripheral groups, aggregation hinders rotation blocking non-radiative decay channels.

Similar trends can be observed in thin films. In Fig. 4, the emission intensities of the films have been corrected by the factor that normalizes their absorption to 1 . It is therefore straightforward to compare the efficiencies of the different compounds as thin films and to detect that the efficiency of 1a film is about 4 (3) times higher than that corresponding to the 1c (1b) film while in solution their efficiencies are almost identical. It should be noted that this material has a strong tendency to self-assembly. In fact upon heating it enters a columnar hexagonal mesophase at $45^{\circ} \mathrm{C}$ that extend in a broad range of temperatures [3]. Another interesting observation is the increase of the red emission efficiency of $\mathbf{1 f}$ thin film compared to its solution (observe the factors applied to the spectra for the solution $(\times 20)$ and for the film $(\times 5)$ in Figs. 2 and 4 , respectively). In this case the behavior of the two emission bands upon the solution concentration is different. While the $413 \mathrm{~nm}$ peak is rapidly quenched following the tendency of the whole series (in fact this emission is not observed in the film) the orange emission is not quenched. Moreover, this orange emission is significantly shifted to the red from the solution to the film (from 551 to $655 \mathrm{~nm}$ ) probably indicating a different configuration of the peripheral groups in the solid state.

\subsection{Fabrication and characterization of organic light emitting diodes}

In order to analyze the semiconducting properties of these materials, solution-processed organic light emitting diodes (OLEDs) based on 1a, 1d and 1e have been fabricated as described in the experimental section. These compounds have been selected considering their electronic properties, specifically their markedly different HOMO levels, band gaps and peak emissions (Table 1), as well as by their observed good film-forming properties. The structure of the fabricated OLED consists of: ITO (140 nm)/PEDOT:PSS $(50 \mathrm{~nm}) /$ active layer/Ca/Al $(200 \mathrm{~nm})$. The thicknesses of the active layers are $85 \mathrm{~nm}, 110 \mathrm{~nm}$ and $116 \mathrm{~nm}$ for 1a, 1d and 1e, respectively.

In Fig. 6 the energy level structure for materials 1a, 1d and 1e, together with the work functions of anode and cathode, are shown. In this plot, we have used the HOMO levels of the solutions and the LUMO level energies calculated using the thin film absorption bandgaps, $E_{g}^{\text {onset }}$ (Table 1) [14]. The work function for PEDOT:PSS thin films has been reported to range from 4.8 to $5.1 \mathrm{eV}$ [15], depending on the PSS content in the top surface upon the addition of high-boiling solvents.

The electroluminescence (EL) spectra recorded from 1a, 1d and 1e-based devices and their evolution with the driving current are plotted in Fig. 7. 1a-based OLED exhibits a deep blue emission, CIE coordinates $(0.16,0.16)$, with the maximum at $427 \mathrm{~nm}$, and well resolved bands at 408 and $439 \mathrm{~nm}$, similar to the thin film PL (Fig. 4). 1d and 1e-based OLED present broad emissions with white (CIE coordinates of $(0.33,0.3)$ ) and whitish green colour (CIE coordinates of $(0.56,0.3))$, respectively. The radiance, at $10 \mathrm{~mA}$, is 0.14 , 0.03 and $0.015 \mathrm{Wsr}^{-1} \mathrm{~m}^{-2}$ for $\mathbf{1 a}, \mathbf{1 e}$ and $\mathbf{1 d}$ respectively. The inferior EL performance of 1d and 1e-based OLEDs is consistent with their lower PL efficiencies. Nevertheless, the recorded luminance for $\mathbf{1 a}, \mathbf{1 e}$ and $\mathbf{1 d}$ are similar for the three devices $\left(9,11.2\right.$ and $3.6 \mathrm{Cd} \mathrm{m}^{-2}$ respectively) because of the low efficiency of human eye response to the deep blue emission of $\mathbf{1 a}(0.03$ at $427 \mathrm{~nm})$.

In devices 1a and 1e a very stable emission with increasing voltage is observed (see normalized spectra in 


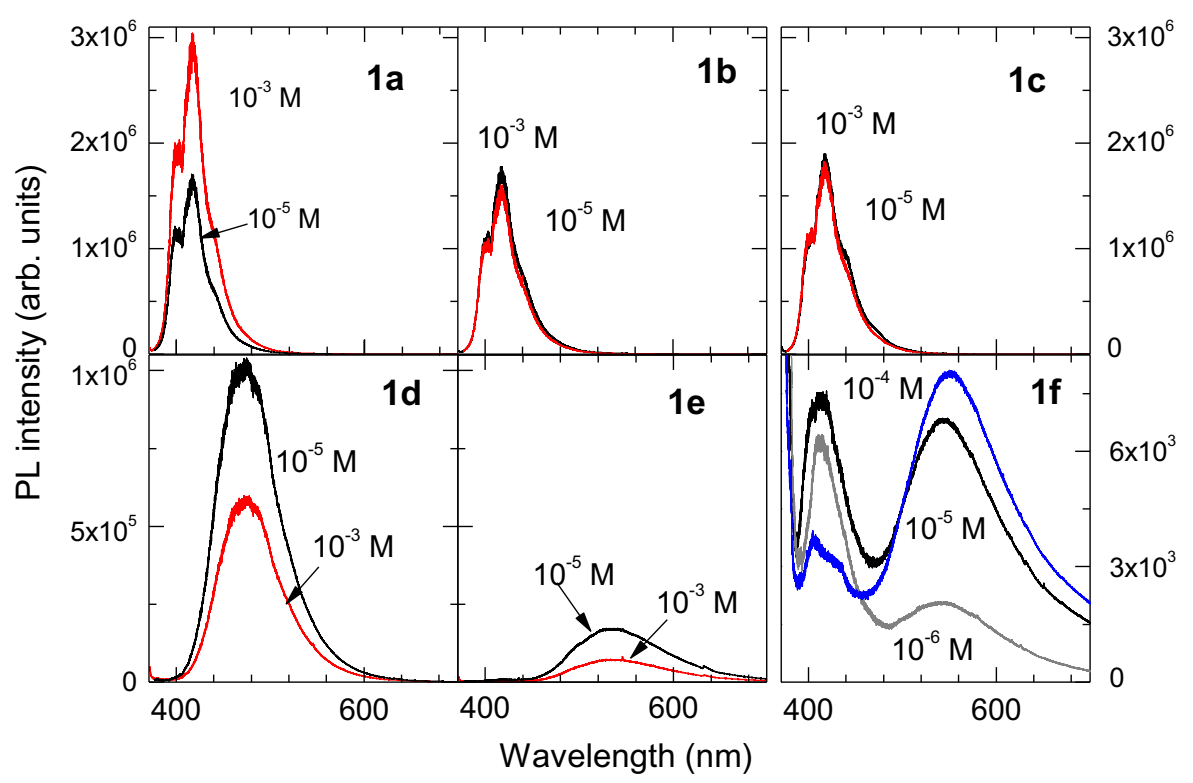

Fig. 5. Photoluminescence, under $365 \mathrm{~nm}$ excitation, of $\mathbf{1 a - 1 f}$ in $\mathrm{CH}_{2} \mathrm{Cl}_{2}$ for different concentrations.

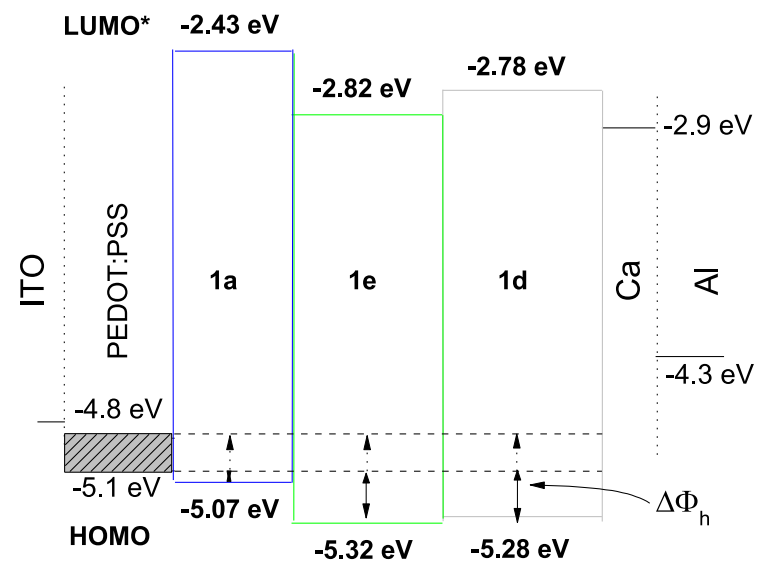

Fig. 7 inset). However, in 1d-based device, with very poor emissive efficiency the noticeable broadening of EL spectra compared to PL is attributed to a degradation which might be associated with an overheating by non radiative relaxation of injected carriers.

In Fig. 8, the experimental $I-V$ responses (open symbols) of 1a, 1d and 1e-based diodes are shown up to currents slightly above the onset for EL. We emphasize that minimizing turn-on voltages, $V_{\mathrm{T}}$, and driving currents is an important issue in the organic electronics industry. In the present case, 1a-based device exhibits a quite low threshold voltage (around $2.8 \mathrm{~V}$ ) together with low driving currents. On the other hand, 1d and 1e-based devices show both higher turn-on voltages $\left(V_{\mathrm{T}}>11 \mathrm{~V}\right)$ and driving currents, while their EL efficiencies are smaller.

The observed EL performance of the three devices may be qualitatively explained in terms of energy level alignment, electrical character ( $\mathrm{p}$ or $\mathrm{n}$ ) of the materials, and charge transport through the device. Using directly the HOMO and LUMO energies of isolated materials to determine the energy level alignment at interfaces is an assumption that may fail at organic-organic or metal-organic junctions, but it provides a reasonable approach for the energy barriers encountered by carriers during transport. On the other hand, in order to estimate the relevance of a barrier, one should keep in mind that for the usual low carrier mobilities in OLED active layers, it has been proven that an injection barrier below $0.3 \mathrm{eV}$ does not limit current significantly [16] since transport turns to be bulk-limited instead of injection-limited. Our experience confirms this statement.

In Fig. 6, the alignment between PEDOT:PSS and 1a levels suggests a negligible barrier for hole injection. According to this view, below $\mathrm{V}_{\mathrm{T}}$, conduction should be carried out by holes. For 1d and 1e materials, the energy level alignment is quite different presenting a noticeable increment of the barrier for hole injection together with a lowering of the electron injection barrier, $(0.12 \mathrm{eV}$ and $0.08 \mathrm{eV}$, respectively) and hence presumably an electron-driving conduction. Nevertheless electron mobility is usually lower than hole mobility in p-type materials. This could explain the higher threshold voltage and the poor EL performance of 1d and 1e-based OLEDs.

To get quantitative insight on the transport and EL mechanisms in the devices we have used a conduction model for voltages below the onset for $\mathrm{EL}, V_{\mathrm{T}}$, where single carrier transport is assumed $[17,18]$ in order to fit the experimental $I-V$ curves (Fig. 8) of 1a, 1d and 1e-based diodes (open symbols). This model takes into account injection barriers, non-uniform electric-field across the layer thickness and field-dependent carrier mobility.

Active layer thickness is a relevant input of the model because it strongly affects the diodes threshold voltage not only in those of bulk-limited conduction but also in 


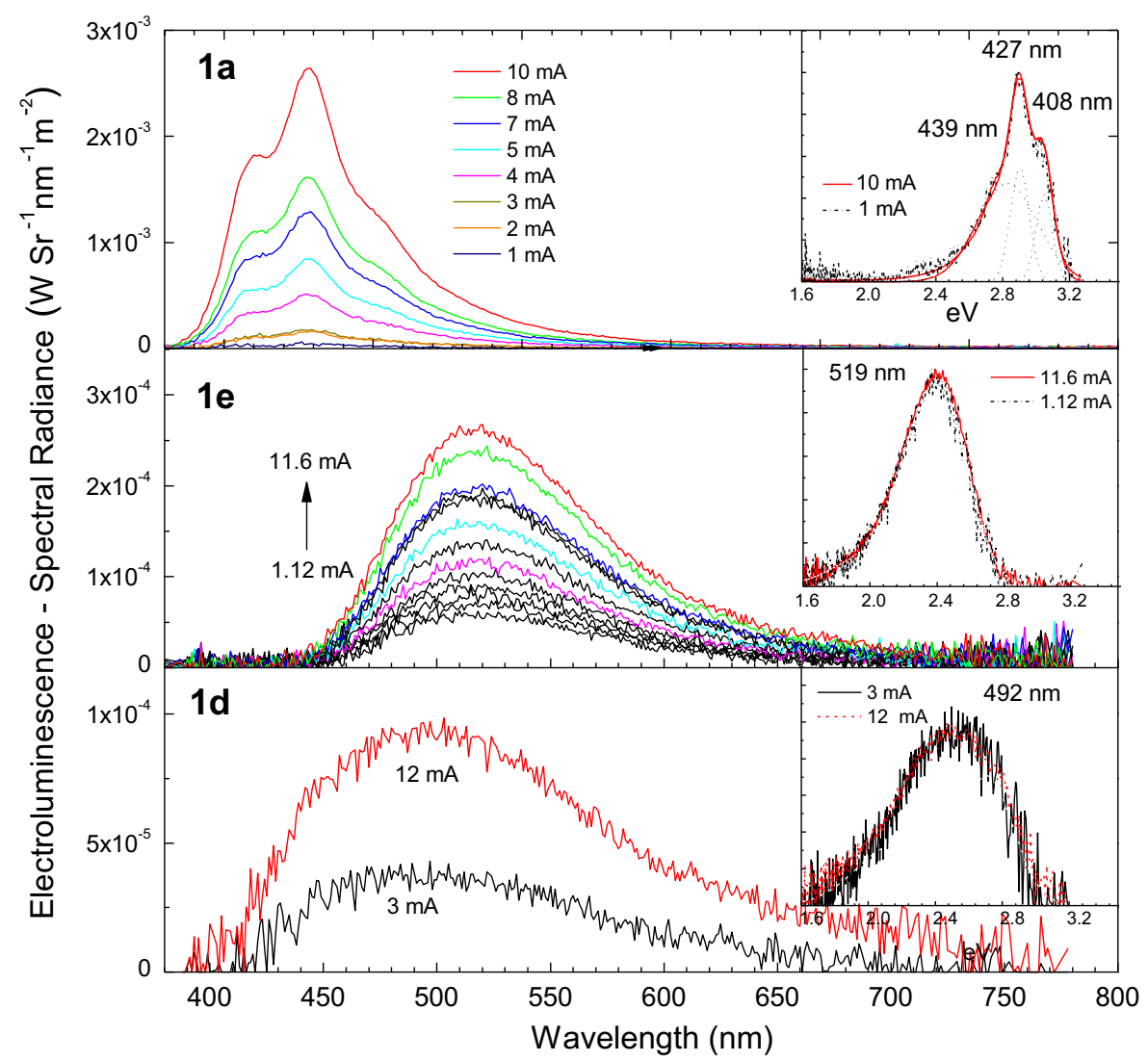

Fig. 7. EL spectral radiance for 1a, 1d and 1e based OLEDs and their evolution with the driving current. The inset shows the normalized EL spectra for the maximum and minimum driving current fitted to a combination of Gaussian functions.

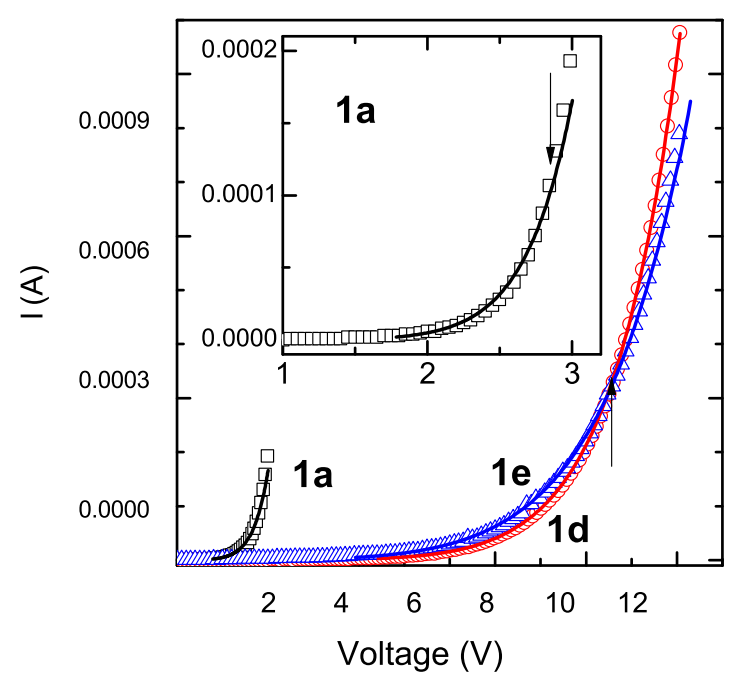

those of injection-limited conduction. In the latter case this is due to the influence of the layer thickness on the interface field which drives the injection process [19]. It is therefore of major concern obtaining the electric field variation across the layer thickness $E(x)$. Moreover, in disordered organic materials carrier transport is usually accomplished by hopping between localized states and the mobility is a field-dependent function that can be approached to the expression $[20,21]$ :

$\mu(E)=\mu_{0} \exp (k \sqrt{E})$

where $E$ is the internal electric field, $\mu_{0}$ is the so-called mobility at zero field [22] (i.e. at $E$ not sufficiently high to enhance the conduction by reducing the effective barrier for trapped carriers) and $k$ is a phenomenological factor related to the lowering of the effective trap depth or Frenkel effect [22]. The expression for the drift current density $J_{b}$ Eq. (2), which includes the mobility Eq. (1), the one-dimensional Poisson Eq. (3) and the integral expression of voltage Eq. (4), are the fundamental equations of the model:

$$
\begin{aligned}
& J_{b}=e \mu_{0} \exp (k \sqrt{E})\left[p_{n}\right] E(x)=e \mu_{0}^{*} \exp (k \sqrt{E[p] E(x))} \\
& \frac{d E(x)}{d x}=\frac{e[p]}{\epsilon \epsilon_{o}} \\
& V_{b}=\int_{0}^{L} E(x) d x
\end{aligned}
$$




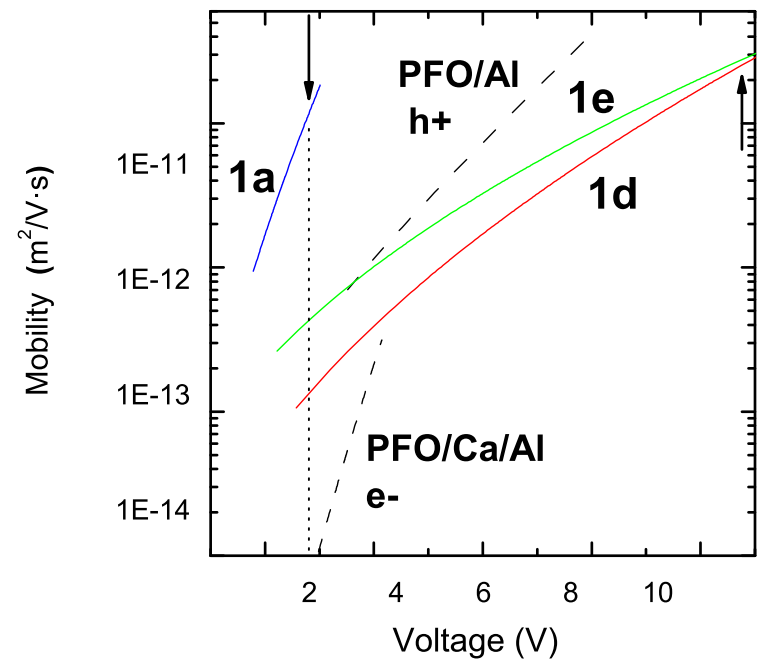

Fig. 9. Carrier mobility $\left(\mu^{*}\right)$-applied voltage relationship derived from the model for 1a, 1d and 1e based OLEDs (continuous lines) and for both PFO devices (dashed lines). The arrows mark the EL onsets.

where $e$ the elementary charge, $\varepsilon_{0}$ the vacuum permittivity, $\varepsilon$ the material dielectric constant, $L$ is the active layer thickness, $V_{b}$ the external bias voltage and $[p]$ is the total carrier density. Finally $\left[p_{n}\right]=\theta \cdot[p]$ is the actual density of carriers contributing to transport. In disordered materials, only a small fraction, $\theta \ll 1$, of the injected carriers, those with energies close to the so-called transport level, contribute to the transport [23]. Since the determination of this parameter is usually difficult, from here on we use the effective mobility $\mu^{*}(E)=\mu(E) \cdot \theta$ and will extract the effective parameter $\mu_{0}^{*}=\mu_{0} \cdot \theta$.

Integrating Eq. (3) using Eq. (2) it is possible to obtain the electric field dependence across the active layer $E(x$, $L, J_{b}$ ). Further details of these calculations have been reported elsewhere $[17,18]$. A second integration of the electric field using Eq. (4) provides the electrical response of the diode $V_{b}\left(E_{0}, E_{L}, J_{b}\right)$ where $E_{0}$ and $E_{L}$ are the values of the electric field at both interfaces: $E_{0}=E(x=0)$, $E_{L}=E(x=L)$. The determination of $E_{0}$ is obtained numerically via the continuity equation for the current density across the device: $J_{\text {inj }}\left(E_{0}\right)=J_{b}$, where $J_{\text {inj }}$ is the injection current which is generally dependent on the electric field at the interface $\left(E_{0}\right)$. In this work we have assumed the injection mechanism proposed by Arkhipov [19], in which the injection of carriers from the Fermi level of the electrode to the conduction level in the organic is not accomplished by conventional tunneling, but by incoherent hopping between localized states with an injection barrier $\Phi$. The described model has been used to fit the experimental $I-V$ curves using a standard quasi-Newton algorithm, where $\mu_{0}^{*}, k$ and $\Phi$ (in $\mathrm{eV}$ ) are the output parameters.

The fits, shown as continuous lines in Fig. 8, reproduce very well the experimental data below the threshold voltage, $V_{\mathrm{T}}$, in all cases while, in those devices with better EL performance (1a and 1e) the agreement gets worse beyond the EL onset. This is attributed to the additional enhancement of conductivity associated with exciton recombination, which is a physical mechanism not included in the model. However, for the 1d-based device, where the EL mediated carrier recombination is quite inefficient (with the worst EL performance), the model still fits nicely beyond this onset.

In order to validate the model and compare the output parameters $\left(k, \mu^{*}\right.$ and $\left.\Phi\right)$ to those of a commercial compound, we have fabricated OLEDs using blue emitting polyfluorene (PFO) with a multilayer structure identical to previous ones, being $L_{\mathrm{PFO}}=85 \mathrm{~nm}$. The PFO HOMO and LUMO levels were elsewhere determined by cyclo-voltammetry to be at $-5.6 \mathrm{eV}$ and $-2.43 \mathrm{eV}$, respectively [24]. To promote an electron-based conduction, a Ca layer was inserted between PFO and $\mathrm{Al}$ at the cathode, whereas the removal of this layer should promote a hole-based conduction (as expected from the Al work function at $-4.3 \mathrm{eV})$. The devices without Ca do not exhibit EL up to $12-15 \mathrm{~V}$, and transport is expected to be driven by holes injected across a noticeable barrier. In the devices with $\mathrm{Ca}$, the onset for EL drops significantly down to $3 \mathrm{~V}$, and electron-driven conduction is assumed. Despite the low threshold voltage, the electron mobility resulting from the model is much lower than that for holes, as shown in Fig. 9. This is consistent with an electron-driven transport in a non-favorable p-type material as this type of polyfluorene [24]. We remark that hole mobility has been extracted in the presence of an injection barrier $\Phi=0.51 \mathrm{eV}$, in reasonable agreement with the energy level scheme.

Table 2 summarizes the obtained fitting parameters, $\mu_{0}^{*}$, $k$, and $\Phi$ for 1a, 1d and 1e based devices and for both PFO OLEDs. Since the electric field $E(x)$ is not uniform, $\mu^{*}[E(x)]$ has been numerically averaged across the whole layer. The resulting average carrier mobility vs. applied voltage $\mu^{*}\left(V_{b}\right)$ is plotted in Fig. 9. We can observe that the carrier mobility in 1a device (Fig. 9) is much higher in its operating range than that for devices $\mathbf{1 d}$ and $\mathbf{1 e}$ (Table 2 collects the average mobilities at $2.8 \mathrm{~V}$ for the all devices).

The present model for charge transport across this OLED structure has allowed us to obtain both electron and hole mobilities for these derivatives by fitting the $I-V$ curves at low voltages $\left(<V_{\mathrm{T}}\right)$ where the transport is dominated by one carrier type. As explained previously, the alignment of the level energies of the successive layers favors hole 
conduction for 1a and PFO/Al devices while mainly electron conduction occurs in 1d, 1e and PFO without Al. The results indicate that hole mobility is more than two orders of magnitude higher compared to electron mobility, which is consistent with p-type materials. This factor is also observed comparing hole and electron mobilities in PFO (Fig. 9 and Table 2). Finally, we emphasize that hole-mobility of the solution processed 1a-based device results significantly higher than that of PFO/Al. Regarding OLED performance, it is clear that increasing the luminance would require the optimization of the electron injection in 1a-based device and of hole injection in 1d and 1e devices. Nevertheless, we can conclude that these solution processed devices exhibited encouraging level of performance in terms of transport.

\section{Conclusions}

We have introduced peripheral groups in the electronrich 10,15-dihydro-5H-diindolo[3,2-a: $\left.3^{\prime}, 2^{\prime}-c\right]$ carbazole (triindole) platform that render solution processable and good film-forming compounds. The influence of the different peripheral groups on the electronic properties of solutions and films is evidenced in the tuning of the HOMO, LUMO, gap and emission energies which are discussed and correlated to the electronic communication of the external substituents with the central electron-rich triindole. The resulting films are amorphous, highly homogeneous and stable for months therefore suitable for device fabrication. The thin films emission ranges from deep blue $(423 \mathrm{~nm})$ to red $(657 \mathrm{~nm})$.

Single layer organic light emitting diodes (OLED) have been fabricated by solution processing methods with the structure: ITO/PEDOT:PSS/active layer/Ca/Al. Three compounds with different HOMO-LUMO alignment to the electrode work functions and different emission wavelengths were used to investigate the influence on transport and emission properties of the substituting species. A numerical model for conductivity that includes injection barriers and non-uniform electric-field across the layer as well as field dependent mobility has been used to analyze the OLEDs $I-V$ response and to compare the results to devices fabricated with commercial PFO. Best results are obtained on the most electron rich derivative functionalized with six donor methoxy groups. This material shows the highest emission efficiency in solid state, due to aggregation-induced enhancement, and favors carrier transport with the highest mobility in the whole operating voltage range. We demonstrated encouraging performance in terms of very low turnon voltage $(2.8 \mathrm{~V})$ and carrier mobility of a solution processed deep blue emitting molecular based OLED.

\section{Acknowledgments}

Support by Comunidad Autónoma de Madrid under project S2009/MAT-1756 and by Ministerio de Ciencia e Innovación (Spain) under projects MAT2009-08786, CTQ2010-18813, TEC2009-13991-C02-02 and TEC201113635-E are gratefully acknowledged.

\section{Appendix A. Supplementary data}

Supplementary data associated with this article can be found, in the online version, at http://dx.doi.org/10.1016/ j.orgel.2012.06.018.

\section{References}

[1] L. Duan, L. Hou, T.W. Lee, J. Qiao, D. Zhang, G. Dong, L. Wang, Y.J. Qiu, Solution processable small molecules for organic light-emitting diodes, J. Mater. Chem. 20 (2010) 6392.

[2] E.M. García-Frutos, E. Gutierrez-Puebla, M.A. Monge, R. Ramírez, P. de Andrés, A. de Andrés, R. Ramírez, B. Gómez-Lor, Crystal structure and charge-transport properties of $\mathrm{N}$-trimethyltriindole: a promising candidate as p-type organic semiconductor, Org. Electron. 10 (2009) 643.

[3] M. Talarico, R. Termine, E.M. García-Frutos, A. Omenat, J.L. Serrano, B. Gómez-Lor, A. Golemme, New electrode-friendly triindole columnar phases with high hole mobility, Chem. Mater. 20 (2008) 6589-6591.

[4] E.M. García-Frutos, U.K. Pandey, R. Termine, A. Omenat, J. Barberá, J.L. Serrano, A. Golemme, B. Gómez-Lor, High charge mobility in discotic liquid-crystalline triindoles: just a core business?, Angew Chem. Int Ed. Engl. 50 (2011) 7399-7402.

[5] R.A. Valentine, A. Whyte, K. Awaga, N. Robertson, New indole trimers as precursors for molecular electronic materials, Tetrahedron Lett 53 (2012) 657-660.

[6] W.Y. Lai, Q.Y. He, R. Zhu, Q.Q. Chen, W. Huang, Kinked star-shaped fluorene/triazatruxene co-oligomer hybrids with enhanced functional properties for high-performance, solution-processed, blue organic light-emitting diodes, Adv. Funct. Mater. 18 (2008) 265-276.

[7] E.M. García-Frutos, G. Hennrich, E. Gutierrez, A. Monge, B. GómezLor, Self-assembly of $C_{3}$-symmetrical hexaaryltriindoles driven by solvophobic and $\mathrm{CH}-\pi$ interactions, J. Org. Chem. 75 (2010) 10701076.

[8] E.M. García-Frutos, A. Omenat, J. Barberá, J.L. Serrano, B. Gómez-Lor Highly ordered p-extended discotic liquid-crystalline triindoles, J. Mater. Chem. 21 (2011) 6831-6836.

[9] E.M. García-Frutos, B. Gómez-Lor, Functionalized triindoles as a new class of p-type organic semiconductors, J. Am. Chem. Soc. 190 (2008) 9173-9177.

[10] C. Coya, A. de Andrés, R. Gómez, C. Seoane, J.L. Segura, On the blue emission of a novel solution-processed stilbenoid dendrimer thin film for OLED displays, J. Lumin. 128 (2008) 761.

[11] Bernard Valeur, Molecular Fluorescence: Principles and Applications, Wiley-VCH, 2001.

[12] The second derivative procedure searches for local minima within a smoothed second derivative data stream. These local minima often reveal hidden peaks. This procedure is often used in commercial software for spectroscopy and chromatography analysis.

[13] Z. Bian, A. Shakouri, Enhanced solid-state thermionic emission in nonplanar heterostructures, Appl. Phys. Lett. 88 (2006) 012102.

[14] For charge injection the relevant energy is the lower edge of the LUMO band which is obtained using the absorption onset.

[15] A.M. Nardes, M. Kemerink, M.M. de Kok, E. Vinken, K. Maturova, R.A.J. Janssen, Conductivity, work function, and environmental stability of PEDOT:PSS thin films treated with sorbitol, Org. Electron. 9 (2008) 727-734.

[16] V.I. Arkhipov, H. von Seggern, E.V. Emelianova, Charge injection versus space-charge-limited current in organic light-emitting diodes, Appl. Phys. Lett. 83 (2003) 5074.

[17] A.L. Alvarez, B. Romero, B. Arredondo, X. Quintana, R. Mallavia, J.M. Oton, Electrical model of organic diodes with field-dependent carrier mobility in the presence of an electric field at the injection interface, Int. J. Numer Model Electron. Networks Devices Fields 23 (2010) 332

[18] C. Coya, A.L. Alvarez, W.S. Yoon, S.Y. Park, Influence of the 1,2,4linking hyperbrancher poly(arylenevinylene) structure on organic light emitting diode performance as compared to convencional 1,3,5-linking one, J. Appl. Phys. 109 (2011) 094507.

[19] V.I. Arkhipov, E.V. Emelianova, Y.H. Tak, H. Bassler, Charge injection into light-emitting diodes: theory and experiment, J. Appl. Phys. 84 (1998) 848.

[20] H. Bässler, Charge transport in disordered organic photoconductors a Monte Carlo simulation study, Phys. Status. Solidi. B 13 (1993) 175

[21] S. Baranosvski, Charge transport in disordered solids with application in electronics, in: S. Baranovski, O. Rubel (Eds.), Wiley, New York, 2006 (Chapter 6). 
[22] P.N. Murgatroyd, Theory of space-charge-limited current enhanced by Frenkel effect, J. Phys. D: Appl. Phys. 3 (1970) 151

[23] V.I. Arkhipov, E.V. Emelianova, H. Bässler, According to the model proposed inside, for a typical carrier localization radius of $2 \AA$, a width of the energetic level of $90 \mathrm{meV}$ and a hopping site density of $1026 \mathrm{~m}^{-3}, \theta$ amounts $2 \times 10^{-7}$, Philos. Mag. B. 81 (2001) 985 .
[24] ]R. Mallavia, F. Montilla, I. Pastor, P. Velasquez, B. Arredondo, A.L. Alvarez, C.R. Mateo, Characterization and side chain manipulation in violet-blue poly-[(9,9-dialkylfluoren-2,7-diyl)-alt-co-(benzen-1,4diyl) backbones, Macromolecules 38 (2005) 3185. 